\title{
Correlation between optoelectronic and structural properties and epilayer thickness of AIN
}

\author{
B. N. Pantha, R. Dahal, M. L. Nakarmi, N. Nepal, J. Li, J. Y. Lin, ${ }^{\text {a) }}$ and H. X. Jiang ${ }^{\text {b) }}$ \\ Department of Physics, Kansas State University, Manhattan, Kansas 66506-2601 \\ Q. S. Paduano and David Weyburne \\ Air Force Research Laboratory, Hanscom AFB, Massachusetts 01731
}

(Received 25 April 2007; accepted 16 May 2007; published online 11 June 2007)

\begin{abstract}
AlN epilayers were grown by metal organic chemical vapor deposition on sapphire substrates. X-ray diffraction measurements revealed that the threading dislocation (TD) density, in particular, the edge TD density, decreases considerably with increasing the epilayer thickness. Photoluminescence results showed that the intensity ratio of the band edge emission to the defect related emission increases linearly with increasing the epilayer thickness. Moreover, the dark current of the fabricated AlN metal-semiconductor-metal deep ultraviolet (DUV) photodetectors decreases drastically with the AIN epilayer thickness. The results suggested that one effective way for attaining DUV optoelectronic devices with improved performance is to increase the thickness of the AlN epilayer template, which results in the reduction of the TD density. () 2007 American Institute of Physics.
\end{abstract} [DOI: $10.1063 / 1.2747662$ ]

AlN has the widest direct band gap $(\sim 6.1 \mathrm{eV})$ among the III-nitrides and possesses outstanding properties such as high temperature stability, high thermal conductivity, and deep ultraviolet (DUV) transparency. These properties make it a good candidate for high power/temperature electronic and optoelectronic device applications such as UV emitters and detectors active in the spectral wavelength region down to $200 \mathrm{~nm}$. AlN epilayers are commonly grown on foreign substrates, such as sapphire and $\mathrm{SiC}$, since high quality AlN bulk wafers are not readily available. Similar with GaN, AlN thin films grown on sapphires are plagued by a high threading dislocation (TD) density on the order of $\sim 10^{9}-10^{10} \mathrm{~cm}^{-2} \cdot{ }^{1-3}$ It is well documented that the presence of high TD density in III-nitrides is a major obstacle for the realization of high performance devices. ${ }^{4,5}$ Thus, approaches for obtaining low defect AlN epilayers as well as effective methods to probe the TD density in AlN are widely sought. Here, we report on the growth and systematic characterization of the optoelectronic and structural properties of AIN epilayers through the measurements of X-ray diffraction (XRD), photoluminescence (PL), and the dark currents of the fabricated photodetectors. The results suggested that one effective way for achieving deep UV optoelectronic devices with improved performance is to increase the thickness of the AIN epilayer template, which results in the reduction of the TD density.

Undoped AlN epilayers were grown on $c$-plane sapphire by metal organic chemical vapor deposition. The sources of $\mathrm{Al}$ and $\mathrm{N}$ were trimethylaluminum (TMAl) and blue ammonia, respectively. We employed DUV PL spectroscopy to investigate the optical properties of AlN epilayers. Metalsemiconductor-metal (MSM) photodetectors were fabricated by depositing Pt $(10 \mathrm{~nm})$ Schottky contacts on AlN epilayers and the fabrication procedure has been outlined in a previous paper. ${ }^{6}$ We used XRD measurements to evaluate the crystal-

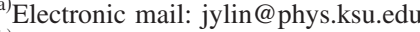

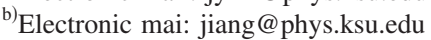

line quality of AlN epilayers. Tilt (out-of-plane rotation) and twist (in-plane rotation) spreads caused by the mosaicity of the thin film were measured and utilized to estimate the density of TDs. $1,7-10$

Rocking curves of $\omega$ scans of the (002) and (102) reflections were measured and the representative results for a $4 \mu \mathrm{m}$ AlN epilayer are illustrated in the inset of Fig. 1. The main plot of Fig. 1 shows the full width at half maximum (FWHM) of rocking curves of $\omega$ scans of the (002) and (102) reflections as a function of the AlN epilayer thickness $t$. The FWHM of the (102) rocking curve sharply decreases with increasing $t$, while that of the (002) rocking curve moderately decreases with increasing $t$. The FWHMs of the (002) and (102) rocking curves as small as 63 and 437 arc sec, respectively, have been measured for the $4 \mu \mathrm{m}$ epilayer, which are among the smallest values reported for AlN epilayers grown on sapphire. ${ }^{11}$

Rocking curves of $\omega$ scans of the (002), (004), and (006) reflections for the $4 \mu \mathrm{m}$ AlN epilayer are shown in Fig. 2(a) and the FWHMs of the (002), (004), and (006) reflections are 63, 72, and 122 arc sec, respectively. We used the FWHM of the (002) reflection to estimate the tilt angle $\beta_{\text {tilt }}$ which was found to be $0.0175^{\circ}$. The density of screw TDs $N_{\text {screw }}$ is then estimated using the classical formula, ${ }^{12}$

$$
N_{\text {screw }}=\beta_{\text {tilt }}^{2} / 4.35 b_{c}^{2},
$$

where $\mathbf{b}_{c}$ is the Burgers vector of $c$-type TDs $\left(\left|b_{c}\right|=0.4982 \mathrm{~nm}\right) . N_{\text {screw }}$ for this film is estimated to be $\sim 5 \times 10^{6} \mathrm{~cm}^{-2}$, which represents one of the lowest reported values for AIN epilayers grown on sapphire.

The twist angle was estimated using the method outlined previously by Lee et al., ${ }^{1}$

$$
\beta=\sqrt{\left(\beta_{\text {tilt }} \cos \chi\right)^{2}+\left(\beta_{\text {twist }} \sin \chi\right)^{2}},
$$

where $\beta_{\text {tilt }}$ and $\beta_{\text {twist }}$ are tilt and twist spreads, respectively, and $\chi$ is the angle between the reciprocal lattice vector $\left(K_{h k l}\right)$ and the (001) surface normal. 


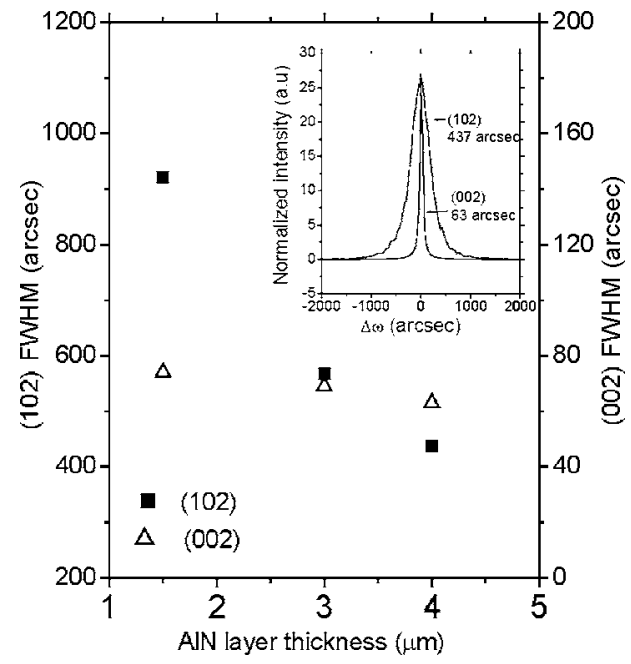

FIG. 1. Epilayer thickness dependence of FWHM of (002) and (102) rocking curves of undoped AlN epilayers. XRD rocking curves of (002) and (102) planes for a $4 \mu \mathrm{m}$ AlN epilayer are shown in the inset.

Figure 2(b) shows the variation of the FWHM of the rocking curves as a function of the inclination angle $\phi$ of lattice plane $(h k l)$ with respect to the $c$ plane. Twist angle was estimated to be $0.188^{\circ}$ by fitting experimental data with Eq. (2). The density of edge TDs $N_{\text {edge }}$ is then calculated using the following classical formula: ${ }^{12}$

$$
N_{\text {edge }}=\beta_{\text {twist }}^{2} / 4.35 b_{a}^{2},
$$

where $\mathbf{b}_{a}$ is the Burgers vector of $a$-type TDs $\left(\left|b_{a}\right|\right.$ $=0.3112 \mathrm{~nm}$ ). The validity of Eq. (3) has already been proven by various authors by comparing the XRD results with those from transmission electron microscopy and etch pit density counting using atomic force microscopy or scanning electron microscopy in GaN. ${ }^{1,8,13} N_{\text {edge }}$ was calculated to be $\sim 2.5 \times 10^{9} \mathrm{~cm}^{-2}$ in the $4 \mu \mathrm{m}$ thick AlN epilayer which is about three orders higher than $N_{\text {screw }}$. The sharp decrease of $N_{\text {edge }}$ with the epilayer thickness indicated by the FWHM of the (102) rocking curve could be due to the termination of an edge type dislocation by forming a loop with similar neighboring dislocations having opposite Burgers vectors as the epilayer thickness increases. The results indicate that AlN epilayer acts as an effective dislocation filter.
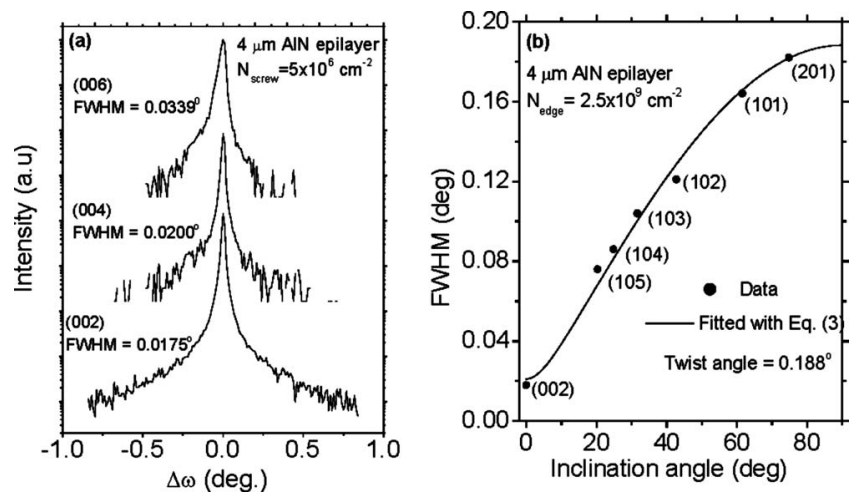

FIG. 2. (a) Rocking curves of $(00 l)$ planes obtained from a $4 \mu \mathrm{m}$ AlN epilayer in symmetric diffraction geometry. (b) Plot of FWHM of $(h k l)$ planes as a function of the lattice plane inclination angle $(\chi)$, according to

Ref. 1 (solid points) and fitted with Eq. (2).
Downloaded 12 Jul 2010 to 129.118 .86 .45 . Redistribution subject to AlP license or copyright; see http://apl.aip.

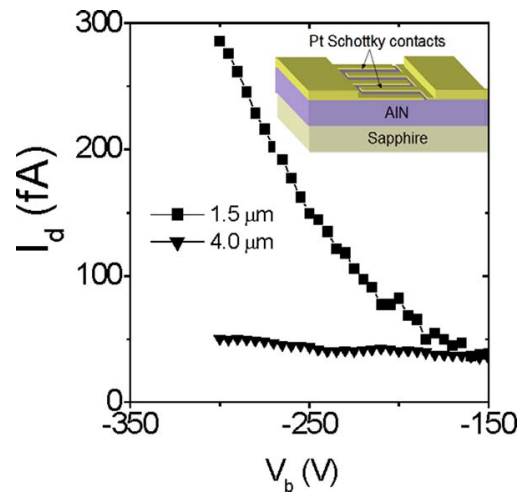

FIG. 3. (Color online) Dark $I$ - $V$ characteristics of AlN MSM photodetectors fabricated from 1.5 and $4 \mu \mathrm{m}$ thick AlN epilayers measured at bias voltages above $150 \mathrm{~V}$. The inset shows the schematic of the device layout (device size $=80 \times 80 \mu \mathrm{m}^{2}$ and finger width $/$ spacing $=2 / 4 \mu \mathrm{m}$ ).

In spite of the recognition of its outstanding physical properties, so far there have been only few demonstrations of AlN as an active DUV optoelectronic device material ${ }^{6,14}$ due to the lack of high quality AlN epilayers in the past. The 1.5 and $4 \mu \mathrm{m}$ AlN epilayers were utilized to fabricate MSM photodetectors with the same active device area. The fabricated AlN MSM photodetectors exhibit a peak responsity at $200 \mathrm{~nm}$, a sharp cutoff wavelength around $207 \mathrm{~nm}$, and more than four orders of magnitude DUV to UV/visible rejection ratio at the applied bias voltages below $150 \mathrm{~V} .{ }^{6}$ Figure 3 shows the dark $I-V$ characteristics of the fabricated AlN MSM photodetectors measured at bias voltages, $V_{b}>150 \mathrm{~V}$. It can be seen that the dark current is drastically reduced in the detector with thick $(4 \mu \mathrm{m})$ AlN active epilayer. The results suggest that the magnitude of the dark current is directly correlated with the density of TDs and DUV photodetectors with improved sensitivity can be obtained by depositing device structures on thick AlN epilayer templates.

The PL emission properties of these AlN epilayers have also been investigated and the PL spectra are displayed in Fig. 4(a), which shows that the emission intensity of the free exciton (FX) recombination $\left(I_{\mathrm{FX}}\right)$ at $5.98 \mathrm{eV}$ (Refs. 15 and 16) increases and that of the deep level impurity line $\left(I_{\mathrm{imp}}\right)$ around $3.8 \mathrm{eV}$ significantly decreases with an increase of the AlN epilayer thickness. More specifically, we noticed from Fig. 4(b) that the intensity ratio of the FX emission to the deep level impurity emission, $I_{\mathrm{FX}} / I_{\mathrm{imp}}$, increases, while the FWHM of the FX transition decreases almost linearly with
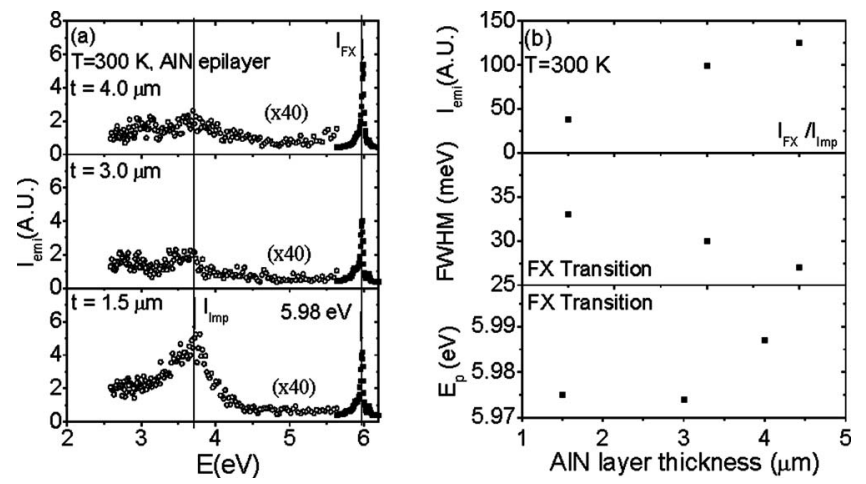

FIG. 4. (a) PL emission spectra of AlN of different epilayer thicknesses. (b) Epilayer thickness dependence of the ratio of the free exciton (FX) emission to the deep level impurity emission $\left(I_{\mathrm{FX}} / I_{\mathrm{imp}}\right)$, the FWHM of the FX transition, and the peak position of the FX transition. 
an increase of the AlN epilayer thickness. Since the optical absorption layer in this wavelength region is on the order of $0.1 \mu \mathrm{m}$, we attribute the increase of the FX emission efficiency and the reduction in FWHM of FX transition to the reduced TD density. The bottom portion of Fig. 4(b) shows that the FX emission peak position blueshifts with an increase of the AlN epilayer thickness, which suggests that the thicker films are more compressively strained.

In summary, structural and optoelectronic properties of undoped AlN epilayers have been studied. For a thick AlN epilayer $(\sim 4 \mu \mathrm{m})$, the FWHM of the rocking curves of the (002) and (102) reflections were as small as 63 and 437 arc sec, respectively, from which $N_{\text {screw }}$ and $N_{\text {edge }}$ for this film were estimated to be $\sim 5 \times 10^{6}$ and $\sim 2.5 \times 10^{9} \mathrm{~cm}^{-2}$, respectively. $200 \mathrm{~nm}$ DUV photodetectors with outstanding characteristics were fabricated from AlN MSM structure and it was shown that the dark current was significantly decreased in photodectectors with thick AlN epilayers. Furthermore, PL measurements revealed that the band edge emission intensity (defect related emission intensity) increases (decreases) with increasing the AlN epilayer thickness. The results suggested that one effective way for attaining DUV optoelectronic devices with improved performance is to increase the thickness of the AlN epilayer template, which results in the reduction of the TD density

This work was supported by ARO and NSF.
${ }^{1}$ S. R. Lee, A. M. West, A. A. Allerman, K. E. Waldrip, D. M. Follstaedt, P. P. Provencio, D. D. Koleske, and C. R. Abernathy, Appl. Phys. Lett. 86, 241904 (2005).

${ }^{2}$ Q. S. Paduano, A. J. Drehman, D. W. Weyburne, J. Kozlowski, J. Seraflnczuk, J. Jasinski, and Z. Liliental-Weber, Phys. Status Solidi C 0, 2014 (2003)

${ }^{3}$ J. Bai, M. Dudley, W. H. Sun, H. M. Wang, and Asif Khan, Appl. Phys. Lett. 88, 051903 (2006).

${ }^{4}$ S. Nakamura and G. Fasol, The Blue Laser Diode: GaN Based Light Emitters and Lasers (Springer, Berlin, 1997), pp. 201-215.

${ }^{5}$ H. Morkoç, Nitride Semiconductors and Devices (Springer, Berlin, 1999), Vol. 32, pp. 149-190.

${ }^{6}$ J. Li, Z. Y. Fan, R. Dahal, M. L. Nakarmi, J. Y. Lin, and H. X. Jiang, Appl. Phys. Lett. 89, 213510 (2006).

${ }^{7}$ V. Srikant, J. S. Speck, and D. R. Clarke, J. Appl. Phys. 82, 4286 (1997).

${ }^{8}$ T. Metzger, R. Höpler, E. Born, O. Ambacher, M. Stutzmann, R. Stömmer, M. Schuster, H. Gobel, S. Christiansen, M. Albrecht, and H. P. Strunk, Philos. Mag. A 77, 1013 (1998).

${ }^{9}$ Y. J. Sun, O. Brandt, T. Y. Liu, A. Trampert, K. H. Ploog, J. Bläsing, and A. Krost, Appl. Phys. Lett. 81, 4928 (2002).

${ }^{10}$ W. R. Busing and H. A. Levy, Acta Crystallogr. 22, 457 (1967).

${ }^{11}$ M. Imura, K. Nakano, N. Fujimoto, N. Okada, K. Balakrishnan, M. Iwaya, S. Kamiyama, H. Amano, I. Akasaki, T. Noro, T. Takagi, and A. Bndoh, Jpn. J. Appl. Phys. 45, 8639 (2006).

${ }^{12}$ C. G. Dunn and E. F. Koch, Acta Metall. 5, 548 (1957).

${ }^{13}$ J. K. Jeong, H. J. Kim, H.-C. Seo, H. J. Kim, E. Yoon, C. S. Hwang, and H. J. Kim, Electrochem. Solid-State Lett. 7, C43 (2004).

${ }^{14}$ Y. Taniyasu, M. Kasu, and T. Makomoto, Nature (London) 441, 325 (2006).

${ }^{15}$ K. B. Nam, J. Li, M. L. Nakarmi, J. Y. Lin, and H. X. Jiang, Appl. Phys. Lett. 82, 1694 (2003).

${ }^{16}$ J. Li, K. B. Nam, M. L. Nakarmi, J. Li, J. Y. Lin, H. X. Jiang, P. Carrier, and S. H. Wei, Appl. Phys. Lett. 83, 5163 (2003). 